\title{
Diseño de procesos basada en riesgo
}

\section{Risk-based process design}

Presentación: 17/10/2019

\section{Doctorando:}

\section{Paola Silvina Biscotti}

CAIMI - FRRo

paola biscotti@yahoo.com.ar

\section{Director/es:}

\section{Nicolás J. Scenna}

\section{Resumen}

En el presente informe se presenta una metodología para el diseño de procesos basada en riesgo y una herramienta informática, llamada CEIBO, que permite aplicarla. También se muestra un caso de estudio donde se aplica CEIBO al diseño de un parque de tanques de almacenamiento.

Palabras Diseño, riesgo, vulnerabilidad.

\begin{abstract}
This report presents a methodology for the design of risk-based processes and a computer tool, called CEIBO, which allows it to be applied. A case study is also shown where CEIBO is applied to the design of a storage tank park.
\end{abstract}

Keywords: Design, risk, vulnerability.

\section{Introducción}

La síntesis o el diseño de un proceso químico es un conjunto de tareas que se deben realizar para desarrollar un proceso que permita obtener un determinado producto. En general, se obtiene como resultado un flowsheet que es la condensación de una estructura de múltiples equipos que se interconectan con el objetivo de transformar las materias primas que ingresan al proceso en los productos deseados. Entre el conjunto de tareas que se deben desarrollar para cumplir con este fin se destacan:

$\checkmark$ Selección de materias primas

$\checkmark$ Selección y ordenamiento de las operaciones y procesos unitarios.

$\checkmark$ Dimensionamiento de cada uno de los equipos y especificaciones de sus condiciones operativas.

El desarrollo de estas tareas de diseño no es trivial ya existen un gran número de alternativas para la selección de los equipos, sus características y su ordenamiento de manera que satisfacer las especificaciones establecidas. Intentar explorar todas las alternativas de diseño es tedioso y por lo general innecesario, generalmente se opta por la comparación de alternativas viables, por ejemplo determinando la factibilidad económica o aplicando reglas heurísticas como guía.

En el diseño o síntesis de procesos "tradicional", la factibilidad económica se utiliza como medida en las primeras etapas del diseño, para seleccionar entre las diversas alternativas factibles y generalmente, la seguridad juega un rol pasivo y se utiliza como un criterio de verificación del diseño final, agregado de niveles de protección en caso de que sea necesario.

Desde hace algunas décadas surge el concepto de "diseño inherentemente seguro", el cual tiene por objeto eliminar el riesgo debido a la operación de procesos, contemplando las potenciales situaciones peligrosas desde las primeras etapas del diseño (Khan y Amyotte, 2002).

Los principios generales del diseño inherentemente seguro son los siguientes (Kletz, 1991):

$\checkmark$ Minimizar: Reducir la cantidad de material peligroso en el proceso

$\checkmark$ Sustituir: Reemplazar un material por otro menos peligroso

$\checkmark$ Moderar: Utilizar condiciones del proceso menos peligrosas tales como presiones y temperaturas más bajas. 
$\checkmark$ Simplificar: Diseñar procesos menos complicados y por lo tanto menos propensos a falla

Se sabe que en la práctica estos principios son difíciles de implementar, a menudo son contradictorios entre sí, y frecuentemente involucran costos crecientes, o directamente imposibilidad tecnológica, por lo que si bien el diseño inherentemente seguro es una referencia, guía o un punto ideal a alcanzar, en muchas situaciones concretas de la práctica ingenieril, una alternativa más realista consiste en definir un objetivo de tolerabilidad para el riesgo (o las consecuencias si se consideran solo éstas) tanto a nivel del personal de la planta como de los pobladores aledaños, y el medio circundante. De esta forma, al considerar la evaluación de la vulnerabilidad del entorno y/o del riesgo en todas las etapas del diseño (incluyendo las preliminares), se pueden descartar aquellas alternativas "intolerables" (según criterios establecidos), durante el propio proceso de diseño y no al final, cuando todas las decisiones (operacionales, logísticas, económicas y financieras) están definidas. En la bibliografía se han propuesto distintas variantes siguiendo esa filosofía, entre ellas la denominada "diseño basado en riesgo" (Rathnayaka et al 2014).

Otra dificultad que se enfrenta es que, en general, realizar un análisis de riesgos no es una tarea sencilla y requiere de mucho tiempo y esfuerzo. La complejidad radica en que se debe contar con información detallada de los equipos de la planta, sus condiciones de operación y su distribución y utilizar modelos matemáticos complejos o software específico para evaluar las consecuencias de un determinado evento. En la etapa de diseño conceptual esto aún es más difícil porque mucha de la información requerida aún no se encuentra disponible.

Para poder realizar la evaluación del riesgo en las primeras etapas del diseño, sin perder rigurosidad en los resultados, es necesario realizar adecuadas simplificaciones. Algunas estrategias propuestas en la bibliografía para tal fin, es decir establecer metodologías de "diseño seguro", se detallan a continuación:

$\checkmark$ Identificar los equipos críticos y centrar el estudio en ellos. Estos equipos son los que tienen una cantidad significativa de alguna sustancia peligrosa o sus condiciones de operación son extremas muy rigurosas (generalmente elevada presión y/o temperatura o muy superiores a las de equilibrio a presión atmosférica).

$\checkmark$ Utilizar modelos sencillos para una evaluación preliminar de las consecuencias de un evento y la vulnerabilidad del entorno. Estos modelos deben permitir una buena aproximación de los resultados involucrando además solo los datos disponibles en la etapa del diseño donde se aplica.

$\checkmark$ Diseñar / Implementar sistemas informáticos adecuados que permitan llevar a cabo los cálculos necesarios en forma eficaz, auxiliando al diseñador durante todas las etapas del diseño.

En general, para la tarea de diseño es habitual utilizar programas de simulación de procesos de acceso libre o bien comercial; como por ejemplo Aspen HYSYS. Por otra parte, si bien existen diferentes softwares que evalúan las consecuencias de un evento peligroso, normalmente, no son compatibles con los programas para simular procesos, tornándose el estudio de riesgo de numerosas alternativas (síntesis de procesos) muy tedioso y complicado, debiendo el usuario prestar atención en no cometer errores en la transcripción de los datos al ingresarlos manualmente en uno y otro software para realizar los cálculos. Esta problemática ha sido resaltada en la bibliografía como uno de los impedimentos operativos más importantes para la práctica de metodologías de diseño basadas en riesgo. En este marco, se propone una metodología y se presenta una herramienta (de desarrollo en CAIMI) para el diseño basado en riesgos, tendiendo a mitigar o resolver las principales limitantes mencionadas más arriba.

\section{Metodología y desarrollo}

En la figura 1 se muestra la metodología propuesta para realizar el diseño de un proceso basado en riesgo. Para comenzar, en base a los objetivos de diseño, y un análisis preliminar de riesgos se adopta una estructura básica o inicial para el proceso (diagrama de flujo del proceso). Luego, este flow-sheet se simula y optimiza para resolver los balances de masa y energía y calcular los principales parámetros de los equipos con las herramientas habitualmente utilizadas para tal fin y se propone la estructura básica de control.

Teniendo en consideración los peligros identificados se propone un layout preliminar y se evalúa la vulnerabilidad del entorno, las distancias de seguridad o el riesgo y se determinan si los mismos resultan aceptables. Por ejemplo, si las distancias evaluadas resultan satisfactorias el layout propuesto se transforma en definitivo sino se realizan modificaciones en la distribución de los equipos y se evalúa nuevamente. En caso de que este procedimiento iterativo no encuentre ningún layout que satisfaga los criterios establecidos para las distancias de afectación o riesgo se deben realizar modificaciones en el diagrama de flujo (por ejemplo, tipo de equipos, número de equipos) o en las condiciones operativas (tales como presión y temperatura) y se continua con el procedimiento iterativamente hasta abordar a un layout definitivo 


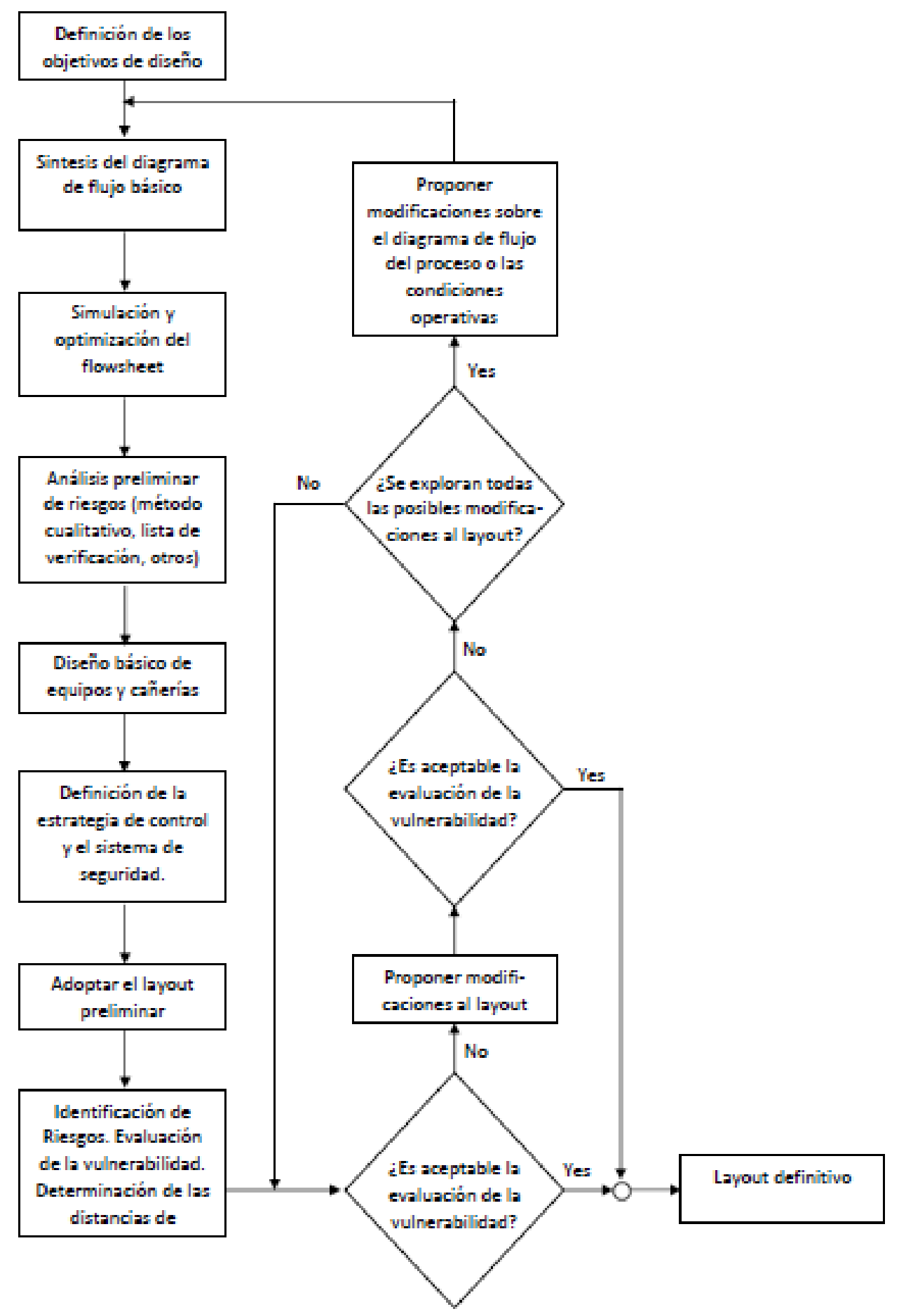

Fig1: Metodología para el diseño basado en riesgo

Para implementar esta metodología se ha trabajado en el desarrollo de un software propio, denominado CEIBO (en continuo desarrollo), el cual cumple múltiples objetivos:

$\checkmark$ Funciona como una interfaz que permite comunicar diferentes softwares, entre ellos simuladores de procesos (como por ejemplo HYSYS), para obtener datos o volcarlos en ellos.

$\checkmark$ Contiene modelos matemáticos para la cuantificación de las consecuencias de distintos tipos de eventos peligrosos

$\checkmark$ Contiene información de árboles de eventos generales para representar las posibles causas - consecuencias a partir de un evento base o iniciador y calcular sus probabilidades

CEIBO cuenta con 3 módulos principales: módulo de cálculo de riesgo, módulo de datos ambientales y un generador de Layout (Figura 2). 


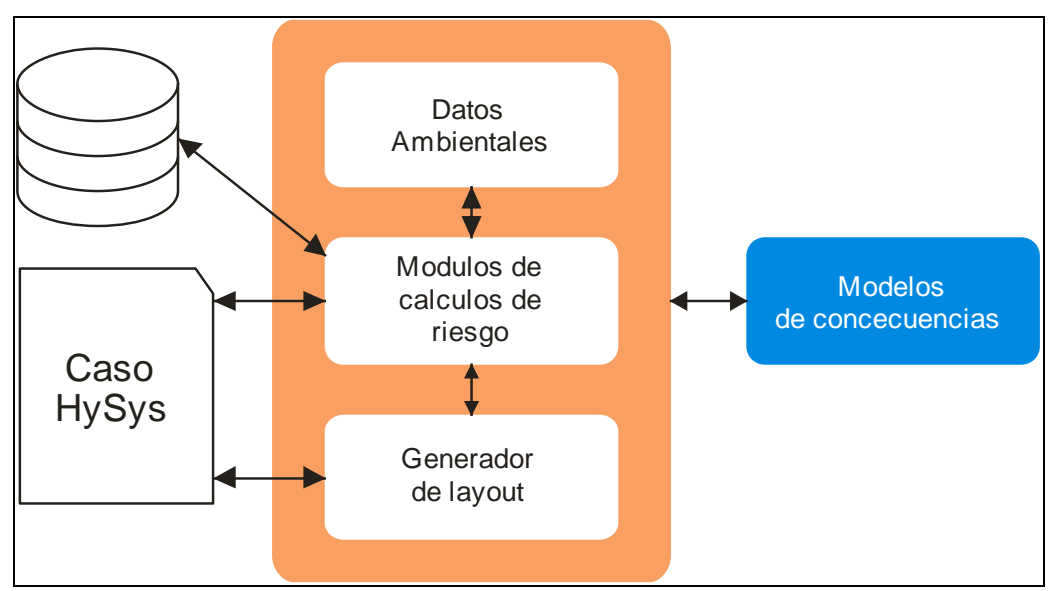

Fig 2: Esquema de módulos que component el software CEIBO

\section{Módulos de datos ambientales}

En este módulo no se realizan cálculos, sólo se ingresan las condiciones ambientales tales como temperatura ambiente y del suelo, humedad relativa o absoluta, presión atmosférica, velocidad y dirección del viento, rugosidad de la superficie; a utilizar por otros módulos de cálculo.

\section{Módulo generador de Layout}

En base a los equipos presentes en el flowsheet (de un caso previamente simulado y convergido en un simulador de procesos), el usuario puede definir la posición que tendrán estos en el terreno.

Toda la información recopilada desde HYSYS, de los diferentes equipos, como así también la información ingresada por el usuario. junto con los datos del terreno donde se encuentra la planta, se puede guardar en archivos, para su posterior reutilización y/o modificación.

Existe la opción de generar un evento en cualquiera de los equipos presentes en el layout para ver cuáles serían sus potenciales consecuencias.

\section{Módulos de cálculo de riesgo}

Este módulo contiene varios submódulos capaces de obtener información necesaria de un caso HYSYS, de generar árboles de eventos (donde se pueden definir las probabilidades de ocurrencia de cada evento, como así también elegir si se simularán todos los escenarios posibles o solo algunos de ellos) y de interactuar con el módulo de datos ambientales, como así también con los demás submódulos de cálculo de consecuencias, basados en los modelos matemáticos propuestos en Guidelines for Chemical Process Quantitative Risk Analysis, AIChE (2000). Si este módulo necesitara más información de la que posee, el usuario puede ingresarla manualmente.

Submódulo Fuga: generalmente una fuga es el comienzo de una situación riesgosa. Este módulo permite calcular el flujo másico, volumen y masa fugados, así como el área del charco formado, a partir de información tal como tipo y propiedades de la/s sustancia/s interviniente/s, nivel de la misma dentro del contenedor, presión, temperatura, diámetro de orificio, tiempo de fuga, entre otras.

Submódulo evaporación: cuando se forma un charco, la sustancia contenida en este puede evaporarse. Este módulo permite estimar la masa evaporada, la velocidad de evaporación y el coeficiente de transferencia de masa, a partir de los datos de la sustancia, ambientales y área del charco.

Submódulo incendio de charco: cuando se forma un charco de una sustancia inflamable, éste se puede incendiar. Éste módulo permite obtener la curva de radiación recibida según la distancia del observador, a partir de información relativa a calor de vaporización, calor de combustión, punto de ebullición, capacidad calorífica, distancia del receptor, datos ambientales (presión, temperatura, velocidad de viento), velocidad fuga, entre otros.

Submódulo incendio jet: cuando se incendia una fuga gaseosa continua, se puede formar una llamarada. Este módulo también permite obtener la curva de radiación recibida según la distancia del observador, a partir de información relativa a calor de vaporización, calor de combustión, punto de ebullición, capacidad calorífica, distancia del receptor, datos ambientales (presión, temperatura, humedad, velocidad de viento), velocidad fuga, entre otros.

Este módulo permite representar las consecuencias en forma gráfica sobre el layout o volcar los resultados en una tabla o una gráfica de intensidad de daño en función de la distancia 


\section{Resultados}

En esta sección se muestra la aplicación del software CEIBO al diseño de un parque de almacenamiento de metanol (materia prima del proceso de producción de biodiesel). Esta selección se basa en el hecho de que en este sector se encuentra el mayor inventario de la sustancia, por lo que a continuación se estudiarán las alternativas de diseño y distribución de los tanques contemplando aspectos de riesgos y cumpliendo las legislaciones vigentes.

Al iniciar el software CEIBO el usuario selecciona el archivo de HYSYS que corresponde al caso de estudio convergido, Luego ingresa al módulo generador de layout para especificar las dimensiones del terreno y la ubicación de los distintos equipos. Este módulo genera un listado con todos los equipos presentes en el archivo de HYSYS. Cada uno de los tipos de equipos tiene una forma predefinida (proyección simplificada).

A continuación, se especifican los datos meteorológicos en el módulo correspondiente.

Para iniciar la simulación de los eventos peligrosos que se desean evaluar se elige el o los equipos donde se van a generar los mismos, en este caso de estudio el/los tanques de metanol, luego se selecciona el evento iniciador para poder asociar el correspondiente árbol de eventos. El usuario puede especificar los tamaños de los agujeros, la ubicación de los mismos y sus probabilidades (o dejar las sugeridas por defecto) Para este caso se asumen que las fugas en cada tanque provienen de orificios ubicados en la base de los mismos, y se simulan dos tamaños de agujeros basados en valores propuestos por la Norma API 581 (API, 2000). Según el árbol de eventos y las propiedades del metanol, los escenarios finales a estudiar corresponden a la posible ignición o no ignición de una fuga líquida (en el caso de ignición, se analiza la posibilidad de incendio del charco o chorro de fuego (Jet-Fire) y la difusión de un gas toxico.

A modo de ejemplo se muestra algunos resultados obtenidos para la evaluación de la intensidad de radiación en función de la distancia al charco de fuego.
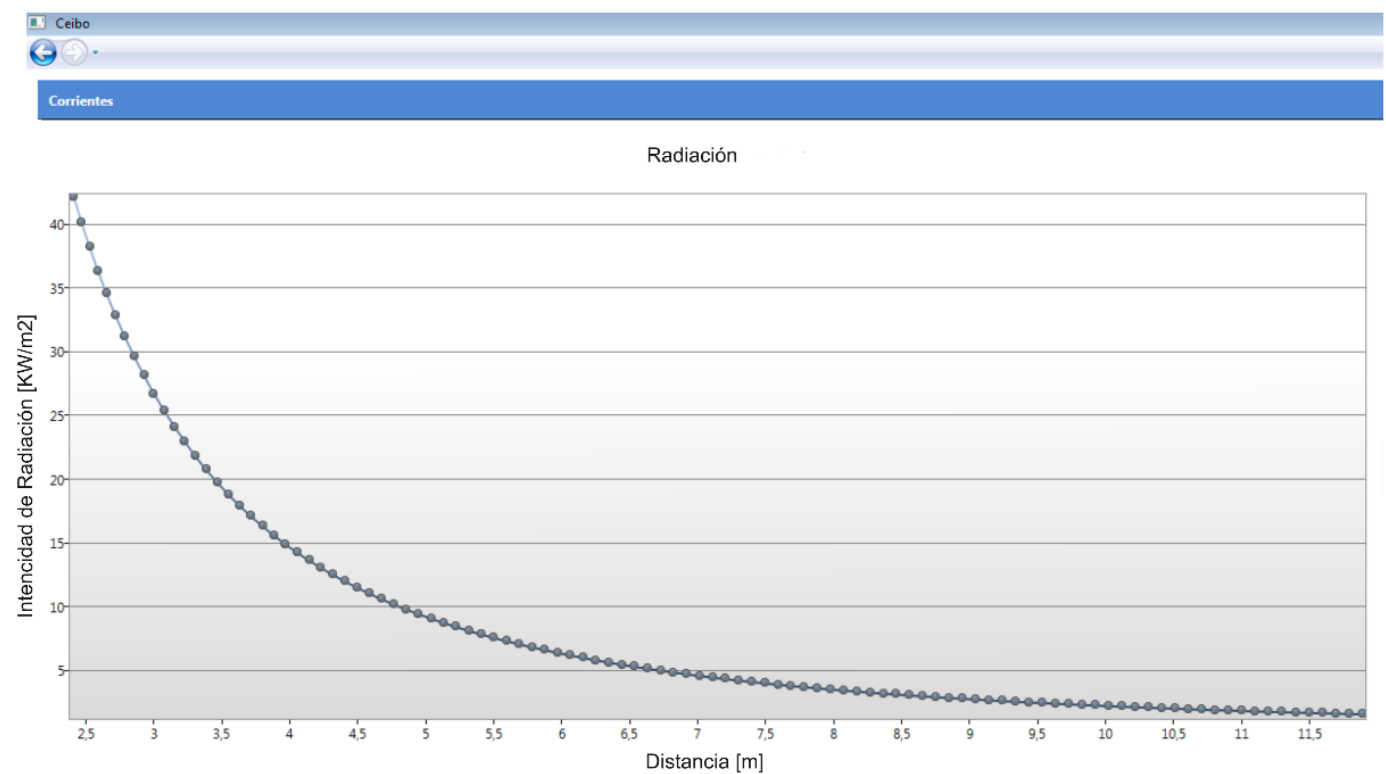

Fig. 3: Intensidad de radiación en función de la distancia

Predefiniendo distintos niveles de interés (por ejemplo, los valores de ERPG) se pueden graficar curvas de isonivel en el layout (figura 4) y evaluar en forma sencilla la posible afectación de otros equipos del layout. En caso de que la distancia de impacto obtenida para los eventos simulados en algún equipo no sea aceptable (por ejemplo, se afecta el exterior del terreno de la planta u otro equipo crítico del proceso) se puede proponer una nueva ubicación en el layout para este ítem. De no encontrarse una ubicación adecuada para este equipo se deben modificar en las especificaciones del caso de HYSYS como por ejemplo temperatura, presión, capacidad del equipo, entre otras y volver a realizar la evaluación de las distancias de impacto sobre el layout con las nuevas condiciones. 


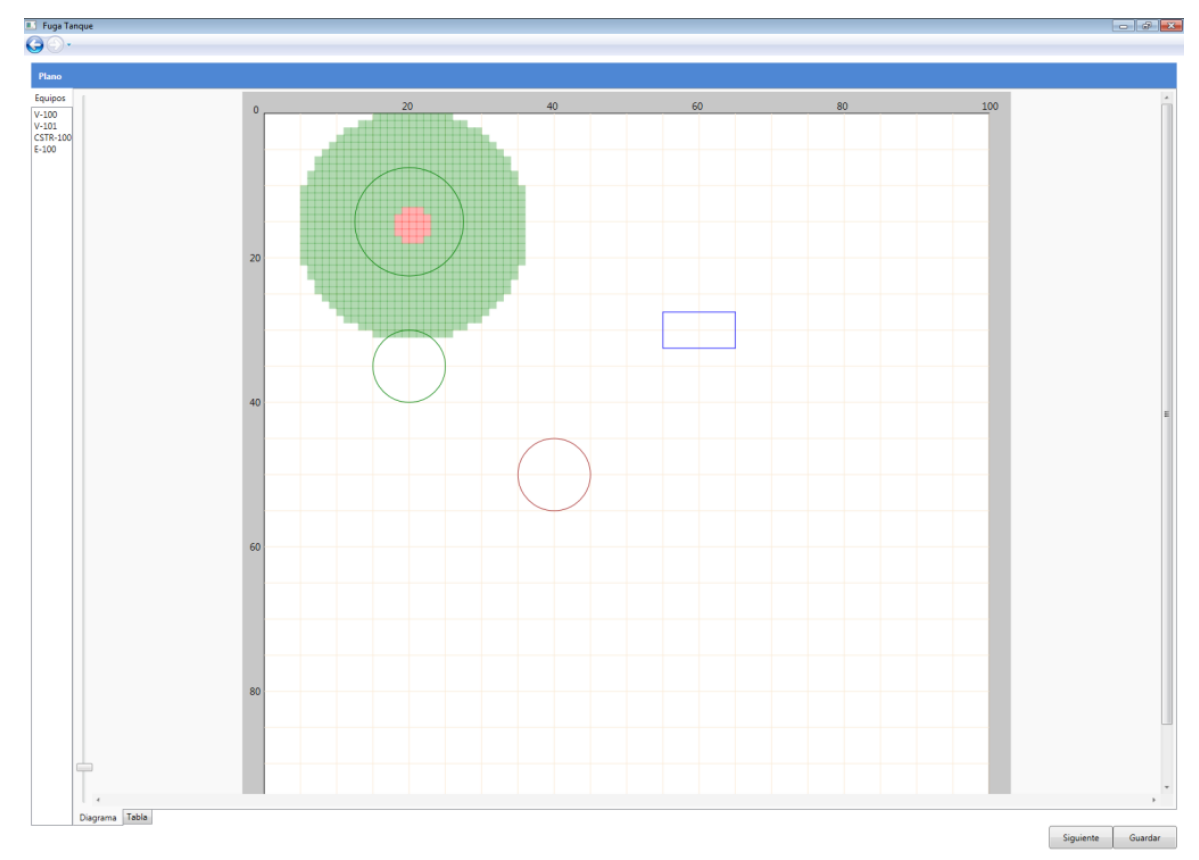

Fig. 4: Distancia de impacto en el módulo generador de layout

\section{Referencias}

AIChE (2000). Guidelines for Chemical Process Quantitative Risk Analysis: American Institute of Chemical Engineers. API 581. (2000). API 581. Washington DC: Americam Petroleum Intitute.

Khan, F. I. \& Amyotte, P. R. (2002). Inherent safety in offshore oil and gas activities: a review of the present status and future directions. Journal of Loss Prevention in the Process Industries, 15, 279-289. doi:10.1016/s09504230(02)00009-8

Kletz, T. A. (1991). Plant design for safety - A user friendly approach. doi:10.1016/0009-2509(92)80335-a

Rathnayaka, S., Khan, F., \& Amyotte, P. (2014). Risk-based process plant design considering inherent safety. Safety Science, 438-464. 